\title{
Die Abhängigkeit der Schneedauer von klimatischen Faktoren in den Berner und Walliser Alpen ${ }^{1}$
}

\author{
Hanspeter Mosimann
}

\section{Begriffe Methoden}

Das Bestimmen der klimatischen Schneegrenze wurde von vielen Autoren mit unterschiedlichen Methoden versucht. Dabei war es leider nicht zu vermeiden, daß der Begriff «klimatische Schneegrenze» verschiedene Inhalte erhielt und sich in verwirrender Vielfalt auf ungleiche Grundlagen und unvergleichbare Gebiete bezog (Messerli 67, S. 197).

In der zugrunde liegenden Arbeit wurden rechnerisch einzelne Punkte von Niveaus bestimmt, welche die Untergrenze der Gebiete markieren, auf denen im Mittel 100, 200 und 365 Tage Schnee liegt. Diese mittleren Grenzlinien werden mit Niveau 100, Niveau 200 und Niveau 365 bezeichnet. Ausgegangen wurde bei diesen Berechnungen von Beobachtungen der permanenten Schneedecke, gemessen in Anzahl Tagen auf horizontalen Feldern.

Zingg (1954) hat die Höhe der klimatischen Schneegrenze in Graubünden berechnet, indem er die gemessene Dauer der Schneedecke als Funktion der Meereshöhe dargestellt hat. Im Zeitraum von 1936 bis 1953 ergibt sich eine mittlere Höhenlage für die klimatische Schneegrenze in Graubünden von $3230 \mathrm{~m}$.

Escher (1969) betrachtet das Temperatur- und Niederschlagsgeschehen als entscheidend für die Dauer der Schneedecke. Er schätzt schließlich die Höhenlage der klimatischen Schneegrenze nur mit der mittleren Jahrestemperatur, da die von ihm als Variable gewählte Jahressumme der Niederschläge gegenüber dem Einfluß der Temperatur ohne wesentliche Bedeutung ist. Die Wahl der Jahrestemperatur stellt einen Kompromiß zwischen der an der Schneegrenze entscheidenden Sommertemperatur und der in der Höhe der Meßstationen den Schneedeckenaufbau beeinflussenden Wintertemperatur dar.

Die gefundene Temperatur-Schneedauer-Relation muß noch in eine Beziehung zwischen Meereshöhe und Schneedauer umgerechnet werden. Escher hat

1 Zusammenfassung einer Diplomarbeit, ausgeführt am Geographischen Institut der Universität Bern. Abteilung Prof. B. Messerli.

die folgenden Höhenlagen für die klimatische Schneegrenze errechnet

$\begin{array}{ll}\text { Graubünden } & 3200 \mathrm{~m} \\ \text { Berner Oberland } & 3200 \mathrm{~m} \\ \text { Wallis } & 3450 \mathrm{~m}\end{array}$

\section{Problemstellung}

Die erwähnten Arbeiten weisen auf eine Reihe von Problemen hin. So ist das Extrapolieren einer gefundenen Regressionsbeziehung bis zu $1000 \mathrm{~m}$ über den Höhenbereich hinaus, in dem die Meßstationen liegen, nur unter günstigen Voraussetzungen möglich. Dies trifft ganz besonders dann zu, wenn die Veränderungen der unabhängigen Variablen, wie zum Beispiel der Temperatur, oberhalb des Meßbereichs nicht vollständig bekannt sind.

Weiter ist die geographische Verteilung der Beobachtungsstationen für das Vorgehen entscheidend, da wir nur bedingt lokal unbeeinflußte Meßwerte zur Verfügung haben. Beobachtungsreihen aus verschiedenen Gebieten werden durch unterschiedliche klimatische Größen beeinflußt. Sie sind deshalb sicher nicht ausschließlich über eine Beziehung zur Meereshöhe oder einer andern Einflußgröße miteinander vergleichbar. Damit wird deutlich, daß für das gewählte Gebiet der Berner und Walliser Alpen, in dem nur ein weitgestreutes Netz von Stationen verfügbar ist, eine einfache Beziehung zwischen Meereshöhe und Schneedauer kaum brauchbare Resultate liefern wird.

Für die Schätzung der Dauer der Schneedecke ist es also sinnvoll, mehrere Klimafaktoren als Variablen heranzuziehen. Deren Verhalten oberhalb der höchsten Meßstationen ist aber, wie erwähnt, nicht vollständig bekannt, so daß nur ein geringes Extrapolieren über den Bereich mit Messungen hinaus vertretbar ist. Aus diesem Grunde sind Variablen ausgesucht worden, welche die Schneeverhältnisse in der Höhenlage, in der Messungen vorliegen, entscheidend beeinflussen. Damit wird also nicht in erster Linie versucht, die Höhe des Niveaus $365 \mathrm{zu}$ bestimmen, sondern wir beschränken uns darauf, die Dauer der periodisch auftretenden Schneedecke zu schätzen und zugleich den Einschnei- und Aus- 

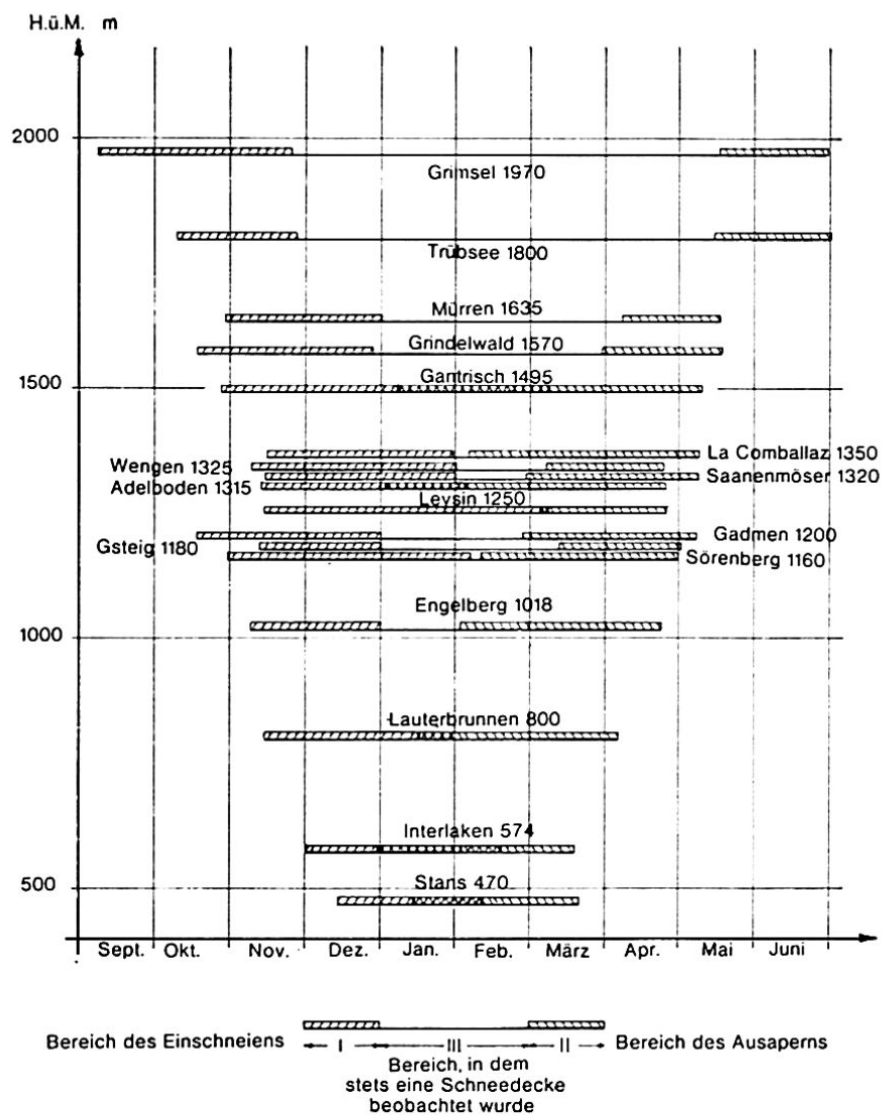

Figur 1a. Einschneien und Ausapern auf der Nordseite der Berner Alpen. Die Balken repräsentieren den Abschnitt, in dem im Beobachtungszeitraum das Einschneien bzw. das Ausapern festgestellt wurde

aperungsvorgang zu untersuchen. Die damit umrissene Aufgabe kann folgendermaßen formuliert werden:

- Welche klimatischen Einflußgrößen sind für die Dauer der Schneedecke entscheidend verantwortlich, und wie variiert dieser Einfluß im betrachteten Gebiet?

- In welcher Höhenlage kann an bestimmten Orten mit 200 Tagen Schneebedeckung gerechnet werden, und wie wird ein Niveau 200 vermutlich im Raume liegen?

- Welche Schwankungen treten beim Einschneien und Ausapern auf, und in welchem Maße verändern sie sich mit der Höhe (Abb. 1)?

Als Einflußgrößen müssen sicher die Temperatur und die Meereshöhe herangezogen werden. Dazu muß noch das Niederschlagsgeschehen berücksichtigt werden. Ich habe als dritte Variable die mittlere Schneehöhe der Monate November bis April gewählt und auf weitere Variablen verzichtet. Dies läßt sich sicher verantworten, da die Schneehöhenangaben zu den Niederschlägen in fester Form die gesamten Auswirkungen des Klimas auf die Schneedecke repräsentieren.

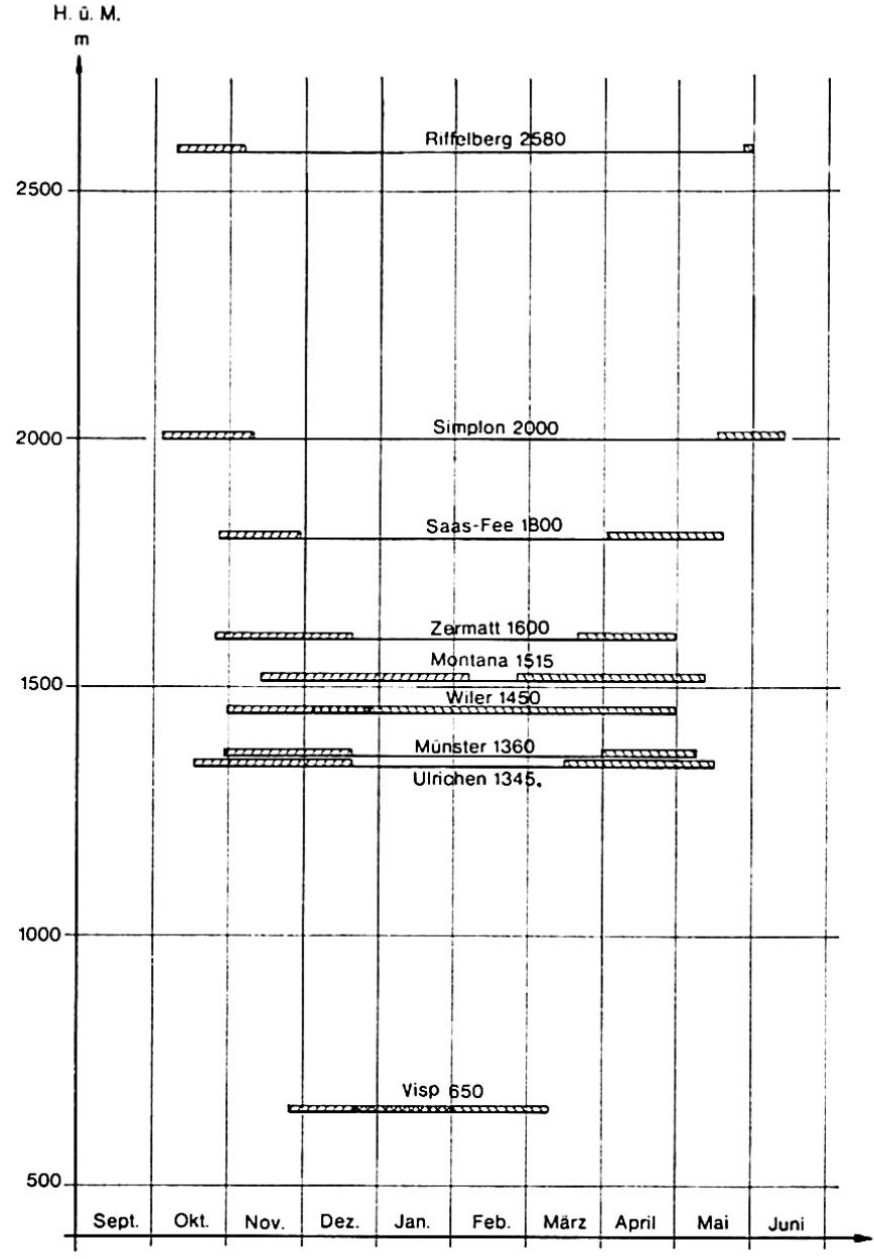

Figur 1b. Einschneien und Ausapern im Wallis (Bei der Station Wiler ist der Winter 1962/63 mit nur 7 Tagen permanenter Schneedecke mitberücksichtigt.)

\section{Diskussion der Daten}

\section{a) Das Stationennetz}

In der Tabelle 1 sind die Stationen aufgeführt, die zur Verfügung standen. Auf der Nordseite ist ihre Verteilung über den Höhenbereich von $500 \mathrm{~m}$ bis $2000 \mathrm{~m}$ relativ gleichmäßig. Im Wallis darf bei der späteren Beurteilung der Resultate nicht unberücksichtigt bleiben, daß mit Visp und Riffelberg zwei Meßstationen in extremer Höhenlage (im Vergleich zu den andern Stationen) vorliegen.

\section{b) Die Schneedauer}

Die Messungen stammen aus den Jahren 1952/53 bis 1968/69 und geben die Anzahl der Tage vom Einschneien der längsten Schneeperiode bis zu deren Ausapern, also die permanente Schneedecke, an. Diese Daten haben den Nachteil, daß bedeutende Fehler bei Stationen unter $700 \mathrm{~m}$ (siehe Fig. 1; Zingg, 1954) auftreten können. Hier wird häufig Schneebedeckung während mehrerer, nicht zusammenhängender Perioden festgestellt. Dagegen sind 
Tabelle 1. Zusammenstellung der Daten über das Einschneien und Ausapern der permanenten Schneedecke

\begin{tabular}{|c|c|c|c|c|c|c|c|c|c|c|c|c|c|c|c|c|c|c|}
\hline 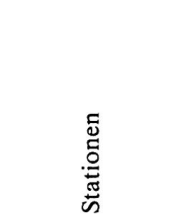 & 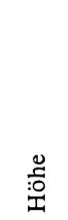 & 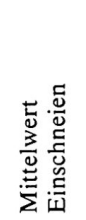 & 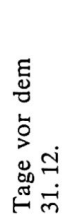 & 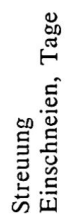 & 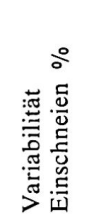 & 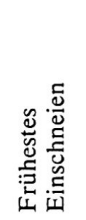 & 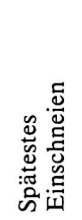 & 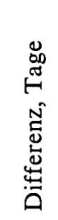 & 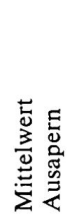 & 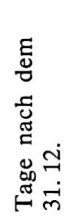 & 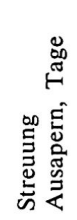 & 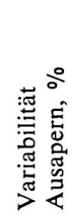 & 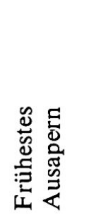 & 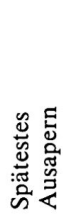 & 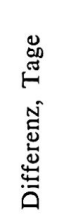 & 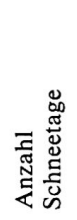 & 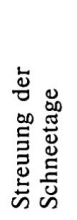 & 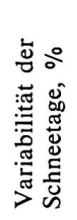 \\
\hline Grimsel & 1970 & 28.10 & 65 & 21,62 & 33,26 & 7. 9. & 25.11 . & 79 & 8.6. & 158 & 15,81 & 10,00 & 15.5 . & 29.6 . & 45 & 226 & 23,27 & 10,29 \\
\hline Gadmen & 1200 & 25.11 . & 37 & 23,71 & 64,08 & 17.10 . & 1. 1 . & 76 & 11.4 . & 100 & 17,92 & 17,92 & 27.2 . & 6.5. & 68 & 138 & 27,42 & 19,87 \\
\hline Trübsee & 1800 & 31.10 . & 62 & 14,17 & 22,86 & 9.10 & 27.11 . & 49 & 11.6. & 161 & 14,79 & 9,18 & 13.5 . & 30.6 . & 47 & 224 & 22,32 & 9,97 \\
\hline Engelberg & 1018 & 6.12 & 26 & 17,8 & 68,45 & 8.11 . & 1. 1 . & 54 & 18.3. & 76 & 22,48 & 29,57 & 2.2 & 22.4 & 79 & 103 & 29,26 & 28,40 \\
\hline Stans & 470 & 6. 1. & -5 & 15,8 & 316,08 & 13.12. & 11. 2. & 60 & 14.2 & 44 & 21,43 & 48,71 & 14.1 . & 19.3 & 64 & 38 & 22,82 & 60,06 \\
\hline Sörenberg & 1160 & 16.12 . & 16 & 29,55 & 184,68 & 29.10 . & 6. 2 . & 100 & 21.3 & 79 & 21,57 & 27,30 & 10.2 . & 29.4 . & 78 & 95 & 38,17 & 40,18 \\
\hline Grindelwald & 1570 & 24.1 & 38 & 20,15 & 53,02 & 17.10 & 27.12 . & 71 & 30.4 & 119 & 13,67 & 11,49 & 29.3 . & 17.5. & 49 & 160 & 18,69 & 11,68 \\
\hline Wengen & 1325 & 10.12 & 22 & 22,01 & 100,06 & 8.11 & 31. 1 . & 84 & 29.3 . & 87 & 16,92 & 19,45 & 6.3 . & 23.4 & 48 & 109 & 33,46 & 30,70 \\
\hline Mürren & 1635 & 28.11 . & 34 & 19,5 & 57,45 & 28.10 & 1. 1 . & 65 & 26.4 . & 115 & 17,33 & 15,67 & 7.4 . & 16.5 & 39 & 154 & 20,84 & 13,53 \\
\hline Lauterbrunnen & 800 & 12.12 & 20 & 20,58 & 102,88 & 14.11. & 29. 1 . & 76 & 3.3. & 61 & 24,78 & 40,63 & 16.1 . & 5.4. & 80 & 81 & 32,86 & 40,56 \\
\hline Interlaken & 574 & 5. 1 . & -4 & 21,29 & 532,28 & 30.11 . & 18. 2 . & 80 & 14.2 . & 44 & 22,11 & 50,24 & 30.12 . & 18.3. & 78 & 40 & 29,69 & 74,24 \\
\hline Adelboden & 1315 & 10.12 . & 22 & 22,03 & 100,15 & 12.11. & 4. 2 . & 84 & 17.3. & 75 & 31,82 & 42,43 & 2.1 . & 23.4 . & 111 & 97 & 37,03 & 38,18 \\
\hline Gsteig & 1180 & 4.12 & 28 & 15,8 & 56,43 & 11.11. & 1. 1. & 51 & 11.4 . & 100 & 16,52 & 16,52 & 11.3. & 1.5. & 52 & 128 & 27,89 & 21,79 \\
\hline Gantrisch & 1495 & 9.12 & 23 & 27,32 & 118,77 & 26.10 . & 6. 3. & 132 & 15.4 & 104 & 28,07 & 26,99 & 7.1. & 8.5. & 121 & 129 & 39,47 & 30,6 \\
\hline Comballaz & 1350 & 10.12 & 22 & 21,16 & 96,2 & 14.11. & 29. 1 . & 79 & 9.4 . & 98 & 23,95 & 24,44 & 5.2 . & 6.5 . & 90 & 120 & 41,93 & 34,94 \\
\hline Leysin & 1250 & 15.12 & 17 & 32,27 & 189,85 & 13.11. & 6. 3. & 113 & 29.3 . & 87 & 17,43 & 20,03 & 4.3. & 22.4 & 49 & 104 & 41,24 & 39,65 \\
\hline Wiler & 1450 & 3.12 . & 29 & 15,88 & 54,76 & 31.10 & 27.12 . & 57 & 3.4 . & 92 & 36,53 & 39,71 & 4.12 . & 30.4 . & 147 & 120 & 35,67 & 29,72 \\
\hline Montana & 1515 & 12.12 & 20 & 22,86 & 114,3 & 13.11. & 7. 2 . & 87 & 11.4 & 100 & 19,35 & 19,35 & 26.2 . & 12.5 . & 75 & 126 & 35,92 & 28,51 \\
\hline Ulrichen & 1345 & 18.11 . & 44 & 18,3 & 41,6 & 17.10 . & 20.12 . & 63 & 23.4 . & 112 & 15,09 & 13,47 & 15.3 . & 15.5 . & 60 & 157 & 22,78 & 14,51 \\
\hline Münster & 1360 & 22.11 & 40 & 15,77 & 39,42 & 29.10 . & 20.12 . & 52 & 20.4 & 109 & 12,78 & 11,72 & 31.3 . & 8.5 . & 38 & 151 & 22,67 & 15,01 \\
\hline Visp & 650 & 25.12 . & 7 & 19,89 & 284,13 & 25.11 . & 31. 1 . & 67 & 3.2 . & 33 & 25,06 & 75,94 & 22.12 . & 9.3 . & 77 & 40 & 28,69 & 71,74 \\
\hline Zermatt & 1600 & 17.11 . & 45 & 16,43 & 36,52 & 25.10 . & 20.12 . & 56 & 12.4 & 101 & 13,95 & 13,81 & 20.3 . & 1.5 . & 41 & 145 & 20,57 & 14,18 \\
\hline Riffelberg & 2580 & 29.10 . & 64 & 8,75 & 13,67 & 9.10 . & 6.11 . & 28 & 29.5 . & 148 & 2,12 & 1,43 & 27.5 . & 1.6. & 5 & 214 & 10,58 & 4,94 \\
\hline Saas-Fee & 1800 & 11.11 . & 51 & 12,19 & 23,9 & 27.10 . & 29.11 . & 33 & 22.4 & 111 & 12,74 & 11,48 & 2.4 & 18.5. & 46 & 163 & 17,45 & 10,7 \\
\hline Simplon & 2000 & 27.10 & 66 & 11,53 & 17,47 & 6.10 & 10.11 & 35 & 27.5 . & 146 & 7,84 & 5,37 & 17.5 . & 13.6. & 26 & 212 & 15,98 & 7,54 \\
\hline
\end{tabular}




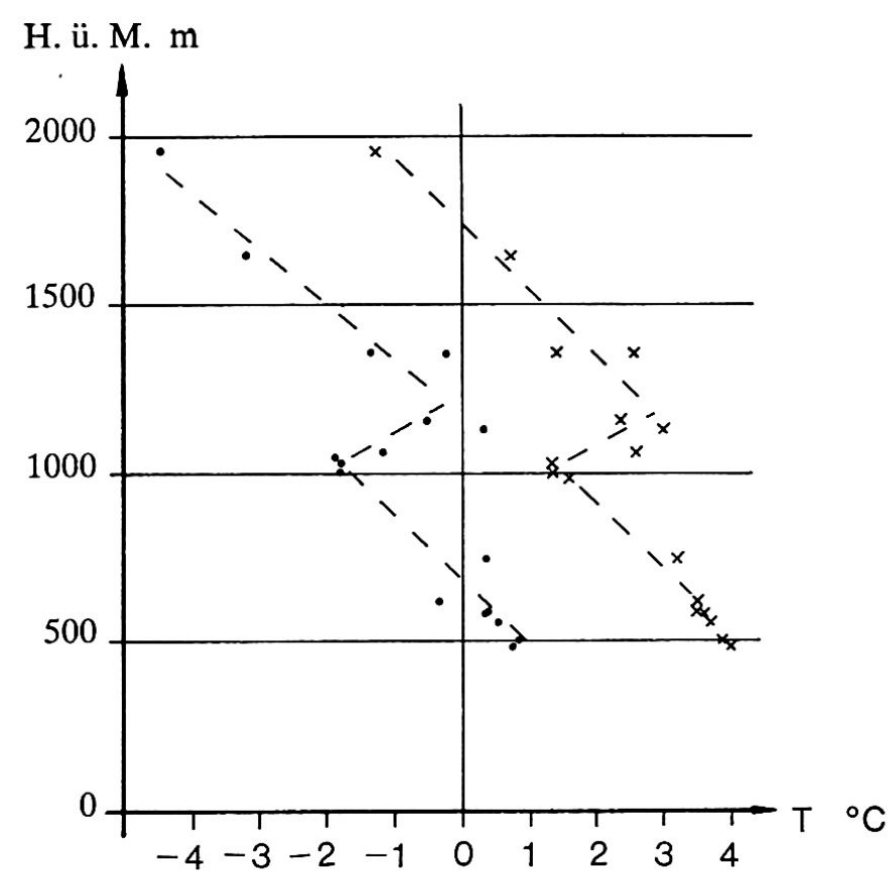

Figur 2. Die Mittelmeertemperatur der Monate November (') und Dezember (x) der MZA-Stationen auf der Nordseite. Die eingezeichneten Linien sind nicht berechnet

bei hochgelegenen Stationen ein paar Einzeltage mit einer Schneedecke, die nur für kurze Zeit liegenbleibt, unbedeutend.

Vorteilhaft wirkt sich für die beabsichtigten Berechnungen sicher aus, daß in allen Höhenlagen ein vergleichbares Ereignis beobachtet wird und unbedeutenden Schneebedeckungen aus einzelnen episodischen Schneefällen nicht das gleiche Gewicht wie der permanenten Schneedecke zugemessen wird. Die Abbildungen 1a und 1b zeigen, wie stark der Beginn und das Ende der permanenten Schneedecke variieren. Dabei ist zu bedenken, daß die Reihen nicht alle vollständig sind, daß also effektiv noch größere Streuungen vorkommen können.

Untereinander direkt vergleichbar sind die Werte der Variabilität (Streuung in Prozenten der Mittelwerte: $100 \mathrm{~s} / \overline{\mathrm{x}}$ ). Die erwähnten Schwierigkeiten beim Festlegen der permanenten Schneedecke in tiefen Lagen führen zu sehr hohen Werten (Interlaken: 532,3) (Tab. 1). Einschneien und Ausapern stehen in einem Zusammenhang mit der Meereshöhe (Zingg, 1954), doch wird diese Beziehung in unterschiedlichem Maße durch Überlagerung anderer Einflußgrößen verändert. Eine besonders deutliche Abweichung zeigt sich im Bereich von $1000 \mathrm{~m}$ bis $1500 \mathrm{~m}$ beim Einschneien. Hier wird, abgesehen von lokal bedingten Differenzen, für den ganzen Höhenbereich das Einschneien in nahezu der gleichen Periode beobachtet. Dieses Phänomen kann zum Teil durch eine in der betreffenden Zeit ziemlich ausgeprägte Inversionsneigung erklärt werden. Figur 2 zeigt die mittleren Temperaturen der
Monate November und Dezember, in denen der betreffende Höhenbereich einschneit. Auffällig ist der Sprung von etwa $1,5^{\circ} \mathrm{C}$ oberhalb $1000 \mathrm{~m}$, der dazu führt, daß die Mitteltemperatur in $1000 \mathrm{~m}$ und $1500 \mathrm{~m}$ ungefähr gleich groß ist. Diese klimatologisch wichtige Größe schafft also im ganzen Höhenbereich ähnliche Bedingungen für den Aufbau der Schneedecke, und das kann zu dem im Mittel gleichzeitig beobachteten Einschneien führen. Die Variabilität der Dauer der Schneedecke zeigt deutlich, daß die Mittelwerte der Meßreihen von Stationen unter etwa $800 \mathrm{~m}$ nur noch eine geringe Aussagekraft besitzen. Damit wird es auch schwierig, die Beobachtungswerte dieser Stationen in eine Beziehung zu Beobachtungen höhergelegener Stationen zu setzen.

\section{c) Die Schneehöhe}

Mörikofer (1948) hat bereits den Zusammenhang zwischen der Meereshöhe und dem Mittelwert der im Winterhalbjahr gemessenen Schneehöhen (mittlere Schneehöhe) festgestellt. Diese Beziehung ist aber nur für Stationen in einzelnen, begrenzten Höhenbereichen angenähert linear. Berechnet man die Regressionsgeraden für jeden Monat mit den Daten der Stationen über $1000 \mathrm{~m}$, so zeigt sich, daß vom November bis in den April der Regressionskoeffizient stetig zunimmt. In größeren Höhen wird die Schneedecke also bis in den Frühling hinein aufgebaut, während in tieferen Lagen der Abbau im Februar einsetzt.

\section{d) Die Temperaturen}

Die jährlichen Werte für jede Station wurden mittels Regressionsrechnung aus den Meßreihen der MZA-Stationen derselben Region berechnet. Dank der im Gegensatz zur Schneedauer und Schneehöhe über größere Gebiete guten Korrelation liefert diese Methode brauchbare Werte. Als Variable zur Schätzung der Schneedauer habe ich die mittlere Temperatur der Monate November bis April (mittlere Wintertemperatur) benützt und damit das Schwergewicht auf die Untersuchung der Schneedauer im Bereiche der Meßstationen gelegt. In der Höhenlage des Niveaus 365 ist die Wintertemperatur für das Überdauern der Schneedecke unwesentlich.

\section{Berechnungen}

Neben den besprochenen Einflußgrößen sind in dieser Arbeit keine weiteren berücksichtigt worden. 
Mit der Methode der Regressionsrechnung sind im Modell

$$
\mathrm{D}=\mathrm{a}+\mathrm{b} \cdot \overline{\mathrm{T}}+\mathrm{c} \cdot \mathrm{S}+\mathrm{d} \cdot \mathrm{H}
$$

mit

$\overline{\mathrm{T}}$ : Mitteltemperatur der Monate November bis April (mittlere Wintertemperatur)

S: Mittlere Schneehöhe vom November bis April

H: Höhe über Meer

D: Dauer der permanenten Schneedecke

die Koeffizienten berechnet worden.

Die Regression mit den Mittelwerten der ganzen Beobachtungsperiode auf der Nordseite der Berner Alpen und im Wallis liefern gute Resultate (Korrelationskoeffizienten: Nordseite: $0,98, \mathrm{n}=$ 17; Wallis: 0,985, $\mathrm{n}=9$ [!]). Entscheidend ist dabei, $\mathrm{da} B$ in beiden Regionen mit der Wintertemperatur die Schneedeckendauer am besten geschätzt werden kann. Das Berücksichtigen der Meereshöhe im Modell bringt bei dieser Variablenkombination keine Verbesserung der Schätzung. Das dürfte mit der Verteilung der Stationen auf verschiedene Täler erklärbar sein. Bei Meßstationen, die alle in einem Tal liegen, kann die Höhe eine wesentliche Einflußgröße für die Schneedauer sein (Zingg, 1954), da die andern Variablen mit der Höhe stark korrelieren. Bei der hier gegebenen Stationsverteilung überlagern aber Einflüsse, die durch Temperatur und Schneehöhe besser repräsentiert werden, die reine Schneedauer-Höhen-Relation.

Die berechneten Werte für die Höhen mit 100 bzw. 200 Tagen Schneebedeckung lauten:

$\begin{array}{lll} & \text { Niveau } 100 & \text { Niveau } 200 \\ \text { Nordseite } & 1120 \mathrm{~m} & 1810 \mathrm{~m} \\ \text { Wallis } & 1250 \mathrm{~m} & 1925 \mathrm{~m}\end{array}$

Legt man der Berechnung die einzelnen Jahresmessungen zugrunde und teilt das ganze Gebiet in kleinere Regionen auf, so resultiert ein bedeutend weniger einheitliches Bild. Die in den Mittelwerten nicht zum Ausdruck kommenden Jahresschwankungen können offenbar nicht überall gleich gut durch den Temperaturverlauf erklärt werden.

Die Abänderung des Modells in

$$
\mathrm{D}=\mathrm{a}+\mathrm{b} \cdot \sqrt{\mathrm{S}}+\mathrm{c} \cdot \overline{\mathrm{T}}+\mathrm{d} \cdot \mathrm{H}
$$

führt wieder zu einer meist übereinstimmenden Rangfolge der Einflußgrößen. Die beste Schätzung der Dauer wird mit der transformierten mittleren Schneehöhe $(S \rightarrow \sqrt{S})$ erreicht.

Eine Untersuchung über die Anwendungsmöglichkeit der Formel hat gezeigt, daß sich auch das
Dezembermittel der Schneehöhe zur Schätzung eignet. Damit wird es möglich, aufgrund des berechneten Dezembermittels eine Aussage über die Schneedauer für das betreffende Jahr zu machen. Das jährliche Mittel der Schneehöhe liefert aber bessere Schätzungen.

Die weitere Unterteilung in Regionen ist eher nach der Zahl der verfügbaren Meßreihen als nach geographischen Überlegungen erfolgt. Nachteilig wirkt sich bei der Regionenbildung der Mangel an Voralpenstationen aus. Erst die Erhöhung ihrer Zahl wird es möglich machen, eine unbedingt notwendige Differenzierung zwischen Voralpengebiet und Alpenraum durchzuführen. Aber auch die Verhältnisse im Wallis sind mit den verfügbaren Stationen nur unzulänglich darstellbar. Gebiete mit einem Eigenverhalten, wie beispielsweise der Simplon, der deutlich unter dem Klimageschehen der Südseite der Alpen steht, können nicht mit genügender Sicherheit abgegrenzt werden.

Tabelle 2. Die berechneten Modelle der einzelnen Regionen und die damit bestimmten Höhenlagen der Niveaus 100, 200 und 365

$$
\begin{array}{ll}
\text { Nordseite total: } & \mathrm{D}=38,7+13,29 \sqrt{\mathrm{S}}-6,59 \overline{\mathrm{T}} \\
\text { Nordseite Ost: } & \mathrm{D}=49,8+11,17 \sqrt{\mathrm{S}}-10,7 \overline{\mathrm{T}} \\
\text { Lütschinentäler: } & \mathrm{D}=17,8+17,32 \sqrt{\mathrm{S}} \\
\text { Nordseite West: } & \mathrm{D}=28,1+15,31 \sqrt{\mathrm{S}} \\
\text { Wallis total: } & \mathrm{D}=34,1+9,67 \sqrt{\mathrm{S}} \\
& -6,47 \mathrm{~T}+0,019 \mathrm{H} \\
\text { Wallis Nord } & \mathrm{D}=22,2+15,32 \sqrt{\mathrm{S}} \\
\text { Oberwallis: } & \mathrm{D}=55,1+9,40 \sqrt{\mathrm{S}}-9,69 \overline{\mathrm{T}} \\
\text { Wallis Süd: } & \mathrm{D}=51,5+11,07 \sqrt{\mathrm{S}}-8,51 \overline{\mathrm{T}}
\end{array}
$$

Die folgende Zusammenstellung gibt die mit diesen Modellen errechneten Höhenlagen der Niveaus 100,200 und 365 wieder.

$\begin{array}{llll} & \begin{array}{l}\text { Niveau } \\ 100\end{array} & \begin{array}{l}\text { Niveau } \\ 200\end{array} & \begin{array}{l}\text { Niveau } \\ \text { Nordseite Ost }\end{array} \\ \text { Lütschinentäler } & 1130 \mathrm{~m} & 1810 \mathrm{~m} & 2900 \mathrm{~m} \\ \text { Nordseite West } & 1120 \mathrm{~m} & 1750 \mathrm{~m} & 2745 \mathrm{~m} \\ \text { Wallis Nord } & 1160 \mathrm{~m} & 1820 \mathrm{~m} & 2950 \mathrm{~m} \\ \text { Oberwallis } & 1190 \mathrm{~m} & 1940 \mathrm{~m} & 2990 \mathrm{~m} \\ \text { Wallis Süd } & 1180 \mathrm{~m} & 2320 \mathrm{~m} & 4100 \mathrm{~m}\end{array}$

Tabelle 2 gibt die berechneten Regressionsgleichungen und die damit bestimmten Meereshöhen für die Niveaus 100, 200 und 365 wieder. Die Höhe für das Niveau 365 wird nur angegeben, um eine Einstufung der Werte zu ermöglichen. Wie fragwürdig das Extrapolieren ist, zeigt sich in den Angaben für die südlichen Walliser Alpen. 


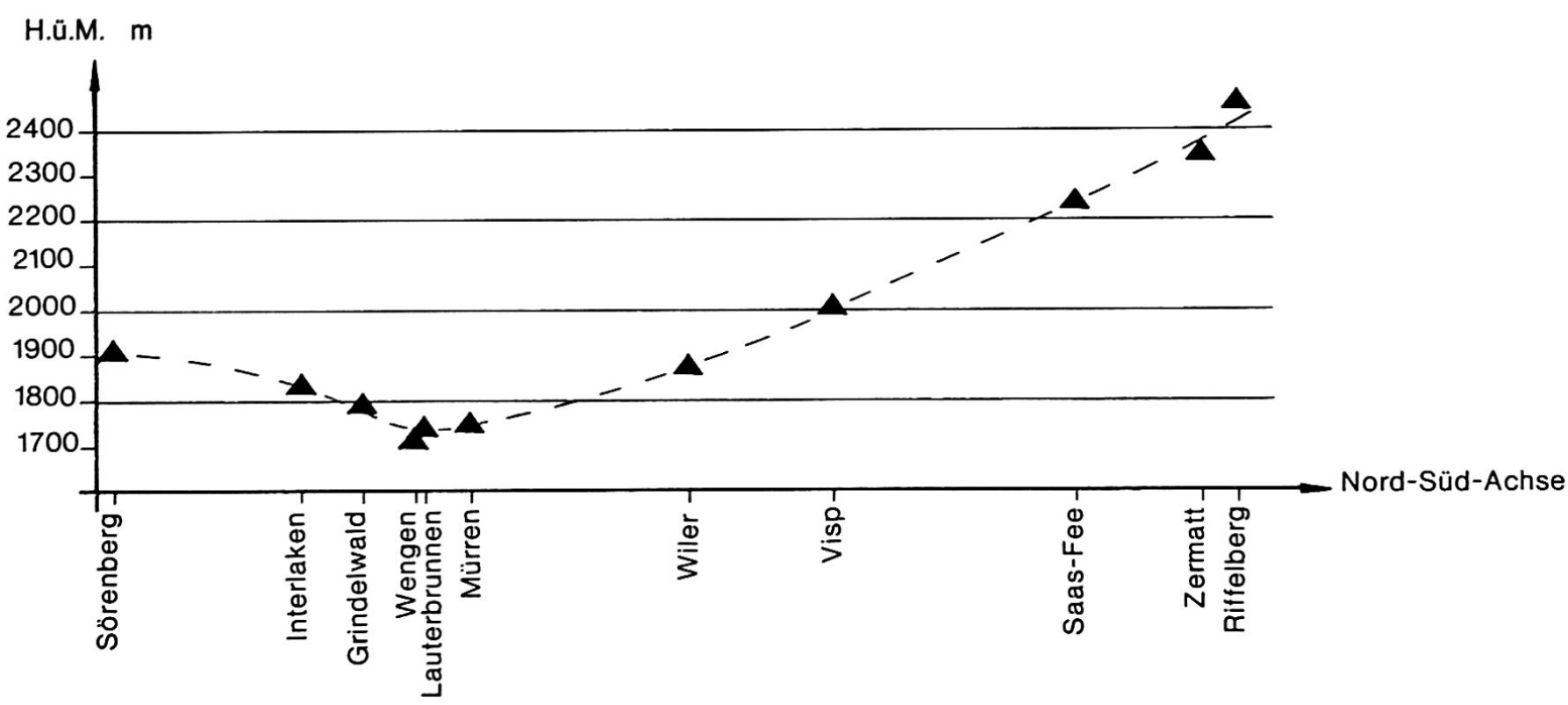

Figur 3. Die Höhenlage des Niveaus 200 bei ausgewählten Stationen längs einer Nord-Süd-Achse. Auffällig ist das Absinken des Niveaus in den Voralpen

Zu den Resultaten sind folgende Úberlegungen anzuführen:

- Auf der Alpennordseite differieren die berechneten Höhen kaum. Der etwas niedrigere Wert für die Lütschinentäler ist auf den Einfluß der Station Interlaken zurückzuführen. In neuen ähnlichen Untersuchungen muß unbedingt berücksichtigt werden, daß tiefliegende Stationen gesondert zu behandeln sind.

- Gegen Süden steigt die Niveaufläche an. Die Resultate in den Visper- und Saasertälern sind nicht auf den ganzen südlichen Walliser Raum ausdehnbar. Die Daten von Riffelberg müssen zuerst überprüft werden. In einer Höhe von $2580 \mathrm{~m}$ ist hier im Mittel die gleiche Dauer wie am Simplon (2000 m, 214 Tage) und eine geringere als auf der Grimsel (1970 m, 226 Tage) gemessen worden. Die geringere Zahl der bei dieser Station verfügbaren Messungen kann dafür nicht verantwortlich sein. Die Frage, ob es sich hier um einen rein lokalen Einfluß handelt oder ob allgemein in dieser Höhe mit besonderen Verhältnissen gerechnet werden muß, kann mit den verfügbaren Unterlagen nicht beantwortet werden. Verantwortlich können die spezielle Lage der Meßstelle (Exposition, Lee-Lage in bezug auf schneebringende Winde, Verluste durch Windverwehungen, Zirkulation usw.) oder eine regelmäßige, hochgelegene Inversion sein. Falls eine Inversion verantwortlich ist, müssen alle bisherigen Berechnungen, die in einem größeren Höhenbereich auf gleichbleibende Gradienten abstellen, neu überprüft werden.
Tabelle 3. Höhe der Niveaus 100, 200 und 365 in Metern über Meer (berechnet)

$\begin{array}{lllc} & \text { Niveau } & \text { Niveau } & \text { Niveau } \\ & 100 & 200 & 365(?) \\ \text { Grimsel } & 1165 & 1845 & 2935 \\ \text { Gadmen } & 1105 & 1790 & 2880 \\ \text { Trübsee } & 1030 & 1715 & 2805 \\ \text { Engelberg } & 1142 & 1825 & 2915 \\ \text { Stans } & 1135 & 1815 & 2905 \\ \text { Sörenberg } & 1228 & 1905 & 2995 \\ \text { Grindelwald } & 1163 & 1790 & 2790 \\ \text { Wengen } & 1075 & 1710 & 2705 \\ \text { Lauterbrunnen } & 1110 & 1740 & 2735 \\ \text { Mürren } & 1115 & 1745 & 2740 \\ \text { Interlaken } & 1210 & 1836 & 2830 \\ \text { Adelboden } & 1130 & 1842 & 2968 \\ \text { Gsteig } & 1040 & 1765 & 2890 \\ \text { Saanenmöser } & 1158 & 1868 & 2990 \\ \text { Gantrisch } & 1108 & 1820 & 2943 \\ \text { La Comballaz } & 1295 & 1995 & 3115 \\ \text { Leysin } & 1150 & 1855 & 2980 \\ \text { Wiler } & 1170 & 1875 & 3000 \\ \text { Montana } & 1182 & 1886 & 3012 \\ \text { Ulrichen } & 1195 & 1940 & 3145 \\ \text { Münster } & 1205 & 1950 & 3155 \\ \text { Visp } & 1265 & 2006 & 3205 \\ \text { Simplon } & 1180 & 1865 & 3130 \\ \text { Zermatt } & 1200 & 2340 & - \\ \text { Saas-Fee } & 1100 & 2235 & - \\ \text { Riffelberg } & 1315 & 2455 & -\end{array}$

Diese Feststellungen haben mich veranlaßt, die Mittelwerte der Variablen jeder Station in die 
Modellgleichung einzusetzen und mittels der Differenz zwischen gemessener und berechneter mittlerer Dauer die Höhe des Niveaus an den Meßstellen zu bestimmen. Die so berechneten Werte (Tab. 3) enthalten natürlich Lokaleinflüsse. Besonders interessant ist hier, daß die Höhen der Niveaus auf der Nordseite der Berner Alpen lokal eine Eintiefung erfahren (Fig. 3).

\section{Zusammenfassung}

In der Arbeit werden die Beziehungen zwischen Schneedauer, Schneehöhe, Meereshöhe und Wintertemperatur untersucht. Dabei wird die Diskussion bewußt auf die Zusammenhänge im Höhenbereich der Meßstationen beschränkt, so daß die wirklich einflußreichen Klimafaktoren festgelegt werden können und die Probleme beim Extrapolieren wegfallen.

Die Untersuchung der Schneedauer im Gebiet der Berner und Walliser Alpen hat gezeigt, daß die Dauer der permanenten Schneedecke in allen Höhenlagen stark variiert. Dafür sind in gleichem Maße der stark ändernde Zeitpunkt des Einschneiens mit Unterschieden von 30 bis 120 Tagen zwischen den einzelnen Jahren und des Ausaperns (30 bis 120 Tage Unterschied) verantwortlich. In Lagen unter etwa $800 \mathrm{~m}$ wird die Streuung sehr groß. Das rührt daher, daß die Schneedecke fast nur episodisch auftritt und deshalb eine permanente Schneedecke nur sehr schwer festzulegen ist (Fig. 1).

Eine eindeutige Höhenabhängigkeit des Einschneiens und Ausaperns wird nur in engbegrenzten Gebieten erfüllt sein. In größeren Räumen überlagern sich andere Einflüsse, die durch Temperatur und Schneehöhe erfaßt werden können. Das Modell zur Schätzung der Schneedauer enthält deshalb diese Variablen neben der Meereshöhe.

Eine Berechnung mit Mittelwerten liefert für die Niveaus 100 und $200 \mathrm{im}$ Wallis Werte, die etwas mehr als $100 \mathrm{~m}$ höher liegen als auf der Nordseite (Nordseite: N 100: 1120 m, N 200: 1810 m; Wallis N 100: $1250 \mathrm{~m}, \mathrm{~N}$ 200: $1925 \mathrm{~m}$ ).

Ein ähnliches Resultat ergibt sich, wenn die Regressionsbeziehung mit den Jahreswerten der Variablen berechnet wird. Hier ist besonders interessant, wie stark die Niveaus im südlichen Wallis ansteigen. Sie liegen hier bis zu $600 \mathrm{~m}$ höher als auf der Alpennordseite. Ungeklärt bleibt, ob diese Werte lokal erklärt werden müssen, oder ob eine hochliegende Inversion allgemein ein lineares Extrapolieren des Modells bis zur Höhenlage des Niveaus 365 fraglich macht. In diesem Falle würde sich natürlich eine Überprüfung aller auf diese Art berechneten Angaben aufdrängen.

\section{Literatur}

Escher, H., 1969: Die Bestimmung der klimatischen Schneegrenze. Diplomarbeit am Geographischen Institut Zürich, unveröffentlicht. Artikel GH Nr. 1, 25. Jg. 1970, S. 35-42.

Fliri, F., 1964: Zur Witterungsklimatologie sommerlicher Schneefälle in den Alpen. "Wetter und Leben».

- 1967: Beiträge zur Kenntnis der Zeit-RaumStruktur des Niederschlags in den Alpen. "Wetter und Leben".

Institut für Schnee- und Lawinenforschung: Winterberichte Weißfluhjoch/Davos, Jahrgänge: 1952/ 53-1968/69.

Jegerlehner, J., 1902: Die Schneegrenze in den Gletschergebieten, Verlag Wilhelm Engelmann, Leipzig.

Messerli, B., 1967: Die eiszeitliche und die gegenwärtige Vergletscherung im Mittelmeerraum, "Geographica Helvetica», Nr. 3, 1967.

Mörikofer, W., 1948: The dependence on altitude of the snow cover in the alps. Extrait des procès verbaux des séances de l'Assemblée Générale d'Oslo (19-28 août 1948) de l'Union Géodésique et Géophysique Internationale.

MZA, 1952-1969: Annalen der Schweizerischen Meteorologischen Zentralanstalt, Zürich.

Uttinger, H., 1951: Zur Höhenunabhängigkeit der Niederschlagsmenge in den Alpen. Archiv für Meteorologie, Geophysik und Bioklimatologie, Bd. II $4 / 1951$.

Zingg, Th., 1954: Die Bestimmung der klimatischen Schneegrenze auf klimatologischer Grundlage, "Angewandte Pflanzensoziologie». Bd. II.

- Mittel- und Extremwerte der Schneehöhe im Versuchsfeld Weißfluhjoch im Zeitraum 1936/37_ $1955 / 56$. Winterbericht 1955/56, S. 123-125.

— Übersicht der Schneeverhältnisse im Gebiet der Schweizer Alpen: - Mittlere und maximale Schneehöhe, Winterbericht 1960/61, S. 137-142. - Neuschneeverhältnisse, Winterbericht 1961/62. Schneeverhältnisse in den Schweizer Alpen, Winterbericht 1965/66, S. 120-128.

\section{Adresse des Verfassers:}

Hanspeter Mosimann

Seminarlehrer

Rohrstraße 48f, 3507 Biglen 